\title{
The Light that Failed - In case of a Patient with Vogt-Koyanagi-Harada Disease
}

\author{
Sur Genel ${ }^{1 *}, 2$, Floca Emanuela ${ }^{1}$, Sur M Lucia ${ }^{1}$, Sur Daniel $\mathbf{G}^{1}$ and Nicula Cristina ${ }^{1,3}$ \\ ${ }^{1}$ University of Medicine and Pharmacy, Iuliu Hatieganu, Cluj-Napoca, Romania \\ ${ }^{2}$ Emergency Clinical Hospital for Children, Cluj-Napoca, Romania \\ ${ }^{3}$ Ophthalmology Clinic, Cluj-Napoca, Romania \\ "Corresponding author: Sur Genel, University of Medicine and Pharmacy, luliu Hatieganu, Cluj-Napoca, Romania, Tel: 0040724504964; E-mail: \\ surgenel@yahoo.com
}

Received Date: Jan 17, 2014, Accepted Date: Mar 24, 2014, Published Date: Mar 27, 2014

Copyright: @ 2014 Sur G, et al. This is an open-access article distributed under the terms of the Creative Commons Attribution License, which permits unrestricted use, distribution, and reproduction in any medium, provided the original author and source are credited.

\begin{abstract}
Although it is not common, impairment of eye in autoimmune inflammatory diseases can become very severe by its evolution and complications. Often eye disorders within the autoimmune inflammatory diseases are not correctly diagnosed. These disorders are usually diagnosed as independent entities and thus local corrective treatment is the only applied. In these situations the evolution of uveitis can be toward blindness. The case presented by us unfortunately fits into this typology of local treatment with repeated surgical interventions without encouraging results. At time of diagnosis the patient completely loses sight in one eye and at the other it dropped to below $50 \%$. In 1907 Rudyard Kipling got the Nobel Prize for his novel" The Light That Failed", whose main character lost view but from other reasons than our patient.
\end{abstract}

Keywords: Uveitis; Autoimmune disease; Corticosteroids

\section{Introduction}

Vogt-Koyanagi-Harada (VKH) disease is a rare disorder characterized by bilateral uveitis with exudative retinal detachments associated or not with cutaneous, neurological, and auditory manifestations. Some researchers have found out that the disease is more common at patients with genetic predisposition to the disease, including those from Asian, Hispanic, Middle Eastern and Native American populations $[1,2]$. The etiology of this disease is not well known, but an immune response to a human leukocyte antigen (HLA) is suspected. This multisystemic disorder is associated with HLA common in autoimmune diseases, includingHLA-DR4, HLA-DR53, and HLA-DQ4. So this disease can be associated with other autoimmune diseases including hypothyroidism, Hashimoto thyroiditis, diabetes mellitus, Guillain-Barré syndrome, and immunoglobulin A nephropathy. It is considered that VKH disease is a cell-mediated autoimmune disease against melanocytes [3].

Because of the multisystemic manifestations of this disease, typical forms are difficult to be diagnosed. The International Committee on Nomenclature established revised criteria for VKH disease diagnosis. According to these criteria three categories of disease were described: complete VKH disease, incomplete VKH disease, and probable VKH disease [4]. The complete form of the disease is characterized by ocular manifestations (diffuse choroiditis with serous retinal detachments or focal areas of subretinal fluid) associated with neurological (meningismus, tinnitus, and cerebrospinal fluid pleocytosis) and skin manifestations (alopecia, poliosis and vitiligo). The incomplete form of the disease is characterized by the presence of either neurological symptoms or cutaneous signs, but not both. Patients with probable VKH disease have only ocular disorder $[5,6]$.

The clinical course of the disease has 4 stages. Prodromal stage, lasting several days, mimics a viral infection. Patients may experience fever, headache, meningismus, nausea, vertigo, orbital pain, and tinnitus. Uveitic stage is characterized by bilateral posterior uveitis, often associated with anterior uveitis. It may last for several weeks. The chronic stage includes ocular and cutaneous manifestations. During this phase, the patient may develop depigmentation of choroid and vitiligo or poliosis. In recurrent stage patients have recurrent or chronic anterior uveitis and ocular complications such as cataract, glaucoma, subretinal fibrosis, and choroidal neovascularization $[6,7]$.

Therapy of this disease consists of early administration of systemic corticosteroids. For patients who do not respond favorably to corticosteroid therapy or who are intolerant to corticosteroids it is recommended immunomodulatory therapy. Intravenous immunoglobulins or biological therapy with infliximab are still under study [8-10].

\section{Case Report}

We present the case of a patient, aged 18 years old, who has been hospitalized in our service for decreased vision in the left eye and right eye vision loss. From history we learned that the patient was born at term with a weight of 2600 grams. At birth, the patient showed no signs of fetal distress and did not require oxygenotherapy or intensive care actions. Psychomotor development was age appropriate, so he attended kindergarten and primary school. When she was 10 , the teacher had found that the girl was having visually impaired, the girl was not seeing the blackboard unless she were to be placed in the first desk. The patient was guided to a doctor but parents have neglected the child and they did not present at ophthalmologic consultation.

Views disorders got worse and after long prevarication patient had consulted an ophthalmologist in a university center. The doctor found the presence of bilateral posterior uveitis. This was more pronounced in the right eye with retinal edema, optic disc hyperemia and edema, and, serous retinal detachments. In the right eye physicians found the presence of anterior and posterior uveitis and synechiae He also noted the presence of cataract and decided cataract surgery. Eye exam 
detected the uveitis in the left eye with retinal edema and optic disc hyperemia. At one time doctors have suspected collagen disease but laboratory examinations that can support the diagnosis of lupus erythematosus and juvenile idiopathic arthritis had been negative. Laboratory analysis showed that anti-DNA antibodies by immunofluorescence are negative and C 3 value is $120 \mathrm{mg} / \mathrm{dl}$. The patient had received local treatments. At the following consultations in ophthalmology service it was found the appearance of secondary glaucoma in the right eye (intraocular pressure of $40-50 \mathrm{mmHg}$ ). It performed surgery, but despite their efforts the patient had lost vision in the right eye and left eye view is greatly diminished. In the left eye inflammation continues its evolution so that synechiae and secondary glaucoma multiply and neoformation vessels arise.

In this condition the patient had been guided in our pediatric service to elucidate a complicated uveitis with unfavorable evolution.

In our service it is suspected the diagnosis of inflammatory disease with ocular manifestations from the first hospitalization. In these circumstances it investigates the existence of a collagen disease, Behcet's disease, Sjogren's syndrome, but the results are negative, infirming these diagnoses (anti-DNA antibodies are negative and the value of C3 is $130 \mathrm{mg} / \mathrm{dl})$.

We resumed history and found that at the age of 8 years the patient presented a rash of skin with erythematous macules and rare vesicles. This event has been interpreted as chickenpox, although the process has not been investigated and proved. So we found that the onset of $\mathrm{VKH}$ disease was at 8 years old in the form of acute infectious episode. Over time untreated disease manifestations leded to ocular complications. It was also found that the girl present skin manifestations of atopic dermatitis on the face with a rough and erythematous leather. This lesion does not mimic the appearance of butterfly found in lupus erythematosus. On physical examination we observed in the elbows and knee the presence of squamous lesions, probably in the context of psoriasis.

Analyzing the clinical aspects mentioned above we interpreted the case with the diagnosis of Vogt-Koyanagi-Harada disease. Neurological examination has revealed no neurological changes. Otorhinolaryngology examination excluded auditory disorders by performing the audiogram and local examination that do not detect pathological changes. The patient has developed incomplete form of the disease because only cutaneous signs were highlighted.

Lumbar puncture was performed for cerebrospinal fluid sampling. Its analysis did not show pleocytosis.

Therapeutic measures included administration of intravenous immunoglobulins at a dose of $5 \mathrm{~g}$ per day for 3 consecutive days and treatment with cortisone at a dose of $1 \mathrm{mg} / \mathrm{kg} /$ day and methotrexate. Treatment with cortisone (prednisone) will have been continued for 4-6 months at minimum effective dose. Methotrextate treatment will be administered for two years. As a therapeutic perspective we took into account biological therapy with Infliximab.

3 weeks after initiation of therapy, ophthalmologic evaluation showed a favorable evolution of posterior uveitis. Ophthalmologic examination showed edema reduction, decreasing the number of neoformation vessels and lack of emergence of new synechiae. In the context of pre-existing glaucoma caused by the synechia existence of anterior and posterior ocular pole, treatment with prednisone led to the increased intraocular pressure. Thus surgical intervention had been performed to correct glaucoma. Because of increased intraocular pressure cortisone administration had been stopped. In these conditions azathioprine was given. The patient continues recommended therapy and the physician follows the evolution of the case.

\section{Particularities of the Case}

1. Onset of the disease at the age of 7-8 years by infectious episode that was not monitored.

2. Skin manifestations appeared sporadically in the beginning, and then as a psoriasis.

3. Unfavorable evolution of ocular manifestations, leading to complications of the disease in the absence osf any treatment other than topical ocular.

\section{References}

1. Damico FM, Bezerra FT, Silva GC, Gasparin F, Yamamoto JH (2009) New insights into Vogt-Koyanagi-Harada disease. Arq Bras Oftalmol 72: 413-420.

2. Greco A, Fusconi M, Gallo A, Turchetta R, Marinelli C, et al. (2013) Vogt-Koyanagi-Harada syndrome. Autoimmun Rev 12: 1033-1038.

3. Attia S, Khochtali S, Kahloun R (2012) Vogt-Koyanagi-Harada disease. Expert Rev Ophthalmol 7: 565-585.

4. da Silva FT, Damico FM, Marin ML, Goldberg AC, Hirata CE, et al. (2009) Revised diagnostic criteria for vogt-koyanagi-harada disease: considerations on the different disease categories. Am J Ophthalmol 147: 339-345.

5. Usui Y, Goto H, Sakai J, Takeuchi M, Usui M, et al. (2009) Presumed Vogt-Koyanagi-Harada disease with unilateral ocular involvement: report of three cases. Graefes Arch Clin Exp Ophthalmol 247: 1127-1132.

6. Arellanes-García LM, Concha-del-Río LE, Preciado-Delgadillo MC (2013) Vogt-Koyanagi-Harada Disease. Retinal and Choroidal Manifestations of Selected Systemic Diseases: 353-376.

7. Sufi Aalia R, Zargar SM, Tejit S (2011) Vogt-Koyanagi-Harada Syndrome. JK Science $13:$ 197-198.

8. Liu X, Yang P, Lin X, Ren X, Zhou H, et al. (2009) Inhibitory effect of Cyclosporin A and corticosteroids on the production of IFN- $\gamma$ and IL-17 by $\mathrm{T}$ cells in Vogt-Koyanagi-Harada syndrome. Clinical Immunology 131: 333-342.

9. Kawaguchi T, Horie S, Bouchenaki N, Ohno-Matsui K, Mochizuki M, et al. (2010) Suboptimal therapy controls clinically apparent disease but not subclinical progression of Vogt-Koyanagi-Harada disease. International Ophthalmology 30: 41-50.

10. Perente I, Utine CA, Cakir H, Kaya V, Tutkun IT, et al.(2009) Management of ocular complications of Vogt-Koyanagi-Harada syndrome. Int Ophthalmol $29: 33-37$. 